\title{
Negative group velocity from quadrupole resonance of plasmonic spheres
}

\author{
Dezhuan Han, Yun Lai, Kin Hung Fung, Zhao-Qing Zhang, and C. T. Chan \\ Department of Physics, The Hong Kong University of Science and Technology, Clear Water Bay, Kowloon, Hong Kong, China
}

(Received 16 December 2008; revised manuscript received 6 April 2009; published 29 May 2009)

\begin{abstract}
We study the dispersion relations of plasmonic bands that arise from the coupling of electric-quadrupole resonances in three-dimensional plasmonic lattices consisting of metallic nanospheres. Through analytical derivation, we show that two branches of quadrupole bands in simple-cubic lattices with a small lattice constant possess negative group velocities. Distinct from double negative $(\varepsilon, \mu<0)$ media in which the negative dispersion originates from the coupling of electric and magnetic responses $(\mathbf{P}$ and $\mathbf{M})$, the negative dispersion induced by quadrupole resonance is an intrinsic property of quadrupole that does not require coupling to another degree of freedom. In addition, the quadrupole dispersions are intrinsically anisotropic, which defies a simple isotropic effective-medium description without spatial dispersion even though the lattice constant is small compared with the wavelength. In plasmonic systems composed of metallic nanoparticle clusters, the coupled quadrupole resonance may be tuned to lower optical frequency and the coupling strength between this quadrupole resonance and external electromagnetic (EM) waves could be in the same order of magnitude as the magnetic dipole $\mathbf{M}$.
\end{abstract}

DOI: 10.1103/PhysRevB.79.195444

PACS number(s): 42.70.Qs, 41.20.Jb, 73.20.Mf

\section{INTRODUCTION}

Negative refraction at a particular frequency range can be achieved if there are simultaneously negative values of electric permittivity $\varepsilon$ and magnetic permeability $\mu .{ }^{1}$ Such kind of material has interesting optical properties, including the possibility of transmitting both propagating and evanescent EM waves. ${ }^{2}$ It is relatively straightforward to obtain negative permittivity, but it is technically difficult to achieve negative permeability especially at optical frequencies. Recently negative refractive index (negative- $n$ ) materials are demonstrated from microwave to optical frequencies by introducing resonant elements such as split-ring resonators (SRR), ${ }^{3}$ and nanoplasmonic structures such as nanorod pairs ${ }^{4}$ and "fishnet" structures..$^{5}$ In optical frequencies, plasmonic clusters such as the ring structure ${ }^{6,7}$ are also plausible candidates to achieve magnetic resonances. In those cases, one have to face the difficulty to define a meaningful magnetic permeability $\mu$, as discussed by Landau, ${ }^{8}$ since the magnetic response in optical frequencies is derived from the curl of electric dipoles. In high frequencies, it may be more convenient to consider the spatial dispersion ${ }^{9}$ of the dielectric function $\varepsilon(\omega, \mathbf{k})$ which can describe comprehensively the electric and magnetic responses. The electric quadrupole $\mathbf{Q}$, generated by the symmetric part of the field gradient, $\operatorname{sym}(\nabla \mathbf{E})$, can be treated as the lowest-order effect which yields the spatial dispersion. Another point which should be emphasized is that, in plasmonic structures such as clusters (ring structure, etc.), $\mathbf{Q}$ is in general of the same order of magnitude as the magnetic moment $\mathbf{M}$. In the parallel metallic nanobar structure, the contribution from $\mathbf{Q}$ has also been proved to be comparable to that from $\mathbf{M}$ in the far field. ${ }^{10}$

In plasmonic lattices, the dispersion can be viewed as the consequence of the hopping of resonances from one unit to another, and in that sense, plasmonic systems are analogous to electrons, where the band structure originates from the hopping of electrons from one site to another in the tightbinding picture. We may thus associate the plasmonic dispersions that are derived from dipoles and quadrupoles with electronic bands of $p$ and $d$ states, respectively. In electronic systems, $s p$ states are usually dispersive and have wide bandwidths, while the $d$ states are more localized leading to a smaller bandwidth. ${ }^{11}$ Similar behaviors are expected in plasmonic systems so that the dipole bands should have a wider bandwidth than the quadrupole bands. In some transition metals, there are strong hybridizations between $s p$ states and the $d$ states. In plasmonics, the hybridization can in principle be tuned as will since the lattices are artificial.

In this paper, we will investigate the dispersion properties of three-dimensional plasmonic lattices made up of small metal nanospheres. Such a problem is typically considered by treating each nanosphere as a dipole. We will give a thorough analysis by including the quadrupoles. It is shown that the quadrupole resonance in simple-cubic lattices can lead to a new kind of negative photonic band which is different from the Veselago type with simultaneously negative values of $\varepsilon(\omega)$ and $\mu(\omega)$. Simple homogenization theories give us permittivity and permeability that depend only on frequency $\omega$, not on wave vector $\mathbf{k}$. The effects of quadrupole cannot be described by such effective parameters if spatial dispersion is not included. It is also different from the Bragg scattering type $^{12}$ since the lattice constant $a$ is much smaller than the wavelength $\lambda$. Compared to the photonic crystal with lattice constant $a \sim \lambda$, the plasmonic systems we deal with are still in the long-wavelength limit. We also note that some authors have shown the negative dispersion for tightly packed plasmonic particles. ${ }^{13}$ However, the mechanism responsible for the negative dispersions, which is the hopping of electric quadrupoles from one site to another in our case, is different from those cases.

This paper is organized as follows. In Sec. II, we calculate the band structures of three-dimensional simple-cubic plasmonic lattices. Bands with negative group velocity are observed independent of the lattice constant $a$, and these negative bands are found to originate from quadrupole resonances. In Sec. III, analytical modeling is developed for quadrupole resonances which is analogous to the coupled dipole equations. ${ }^{14,15}$ The negative group velocities $v_{g}$ are derived analytically and the anisotropy of the bands are found even in the long-wavelength limit. In Sec. IV, we fur- 

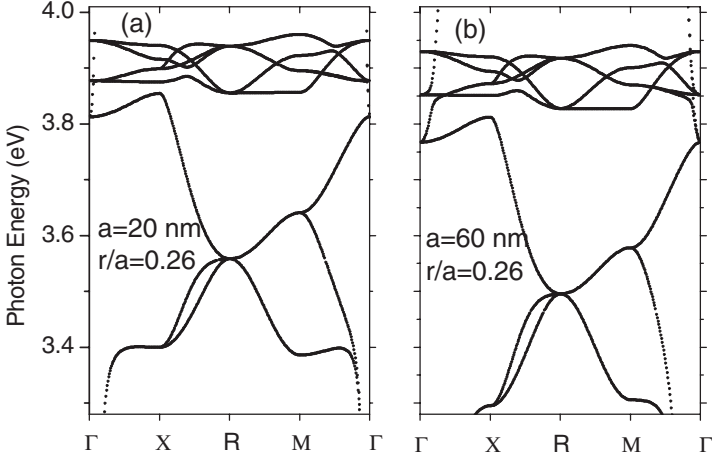

FIG. 1. Band structures of simple-cubic plasmonic lattice calculated by MST with the ratio $r_{s} / a=0.26$ fixed. The lattice constants in (a) and (b) are 20 and $60 \mathrm{~nm}$, respectively.

ther consider the plasmonic dispersion for the quadrupole mode of a nanoparticle cluster, using an octahedral cluster as prototype. We conclude this work in Sec. V and in the Appendix we give mathematics details that are omitted from main text.

\section{QUADRUPOLE BANDS}

We first calculate the photonic band structures of simplecubic plasmonic lattices by solving the Maxwell equations using the multiple-scattering theory (MST) ${ }^{16}$ We note that MST for spherical objects is based on the expansion of the electric and magnetic fields in terms of vector spherical harmonics, and the convergence is controlled by the number of angular-momentum channels used. If the calculation is truncated so that only spherical harmonics with $l=1$ are used, the results will be equivalent to using a dipole approximation, while if only $l=2$ terms are used, it is a quadrupole approximation. It is known in the literature that for metallic spheres arranged in one dimensional chains, the dipole and quadruple dispersions are separated in energy if $r_{s} \leq a / 3 .{ }^{17} \mathrm{In}$ this paper, we use the Drude-type permittivity $\varepsilon(\omega)=1-\omega_{p}^{2} / \omega^{2}$ to describe the response of the metal with a bulk plasma frequency of $\omega_{p}=6.18 \mathrm{eV}$. The salient features of the results remain the same for other choices of the plasma frequency. In Fig. 1, we show the band structures for two values of the lattice constant $a$ (20 and $60 \mathrm{~nm}$ ) while keeping the ratio of the radius to lattice constant $r_{s} / a=0.26$ fixed. We find a total of eight bands, three of them are of dipole origin, and the other five are classified as quadrupole bands. Counting the bands from low energy to higher energy, the two low-lying bands are transverse dipole bands, in which the dipoles are perpendicular to the $\mathbf{k}$-vector. We then have the longitudinal dipole band at higher energy, with the dipoles pointing along the $\mathbf{k}$-vector. The five quadrupole bands near $3.9 \mathrm{eV}$ are less dispersive. Bands with negative group velocity near the zone center are observed for two branches of the quadrupole bands. The calculations show that the band structures with $l=1,2$ have only very slight modifications if higher multipoles $l \geq 3$ are included. This is not surprising if we note that the quadrupole bands do not reach the octupole $(l=3)$ resonance frequency $\omega_{l=3} \sim \sqrt{\frac{l}{2 l+1}} \omega_{p}=4.05 \mathrm{eV}$. The quadrupole
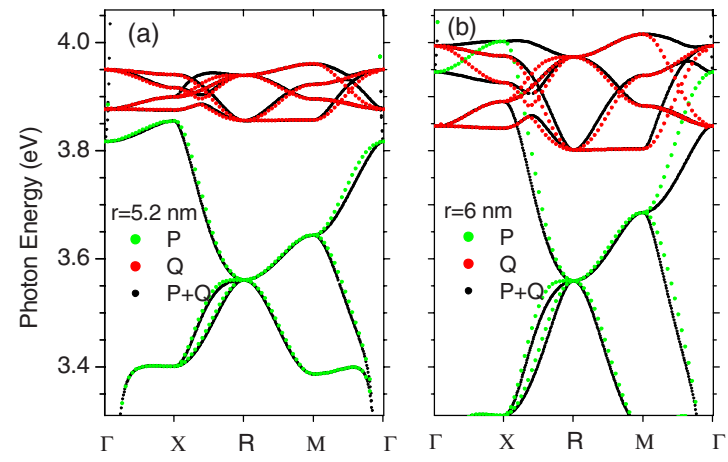

FIG. 2. (Color online) Band structures calculated by MST with $l=1,2$ (black dots), and $l=1, l=2$ separately shown as light gray (green) and dark gray (red) dots. The sphere radii $r_{s}$ in (a) and (b) are 5.2 and $6 \mathrm{~nm}$, respectively, while the lattice constant $a$ $=20 \mathrm{~nm}$ is fixed.

bands are found to be essentially scale invariant, in the sense that the dispersion is nearly the same in an absolute frequency scale if we vary the lattice constant but keep the ratio $r_{s} / a$ fixed. However, a small redshift of about $0.02 \mathrm{eV}$ in photon energy are observed in Fig. 1 when the sphere radius increases from 5.2 to $15.6 \mathrm{~nm}$. This redshift comes from the redshift of the single sphere resonance frequency when the sphere radius becomes larger. ${ }^{15}$ The longitudinal dipole mode is also very robust, while the transverse modes change more conspicuously because they are coupled to free photons whose dispersion is given by the light line, which move closer and closer to vertical axis with the slope proportional to $\pi / a$ as the lattice constant becomes smaller. As a consequence, the dispersion of the transverse dipole modes have a larger slope when $a$ becomes smaller as shown in Fig. 1 . Similar phenomena can be observed for longitudinal and quadrupole modes near 3.8 and $3.9 \mathrm{eV}$ as the light line intersects the plasmonic resonances.

Let us now consider the hybridization of the $\mathbf{P}$ and $\mathbf{Q}$ modes. The polarizability $\alpha_{p}$ and $\alpha_{Q}$ for $\mathbf{P}$ and $\mathbf{Q}$ are proportional to the corresponding Mie coefficients ${ }^{18}$ such that $\alpha_{p}(\omega)=3 i a_{1}(\omega) / 2 k_{0}^{3}$, and $\alpha_{Q}(\omega)=30 i a_{2}(\omega) / k_{0}^{5}$, where $k_{0}$ $=\omega / c$ is the wave vector in vacuum. By setting $\alpha_{p}=0$ (or $\alpha_{Q}=0$ ), we can obtain a "pure quadrupole" or "pure dipole" dispersion, respectively. Figure 2 compares the complete band structure (black), which treats all angular-momentum channels on equal footing, with pure dipole and pure quadrupole bands shown by light gray (green) and dark gray (red) dots, respectively. We can see that for the same lattice constant, the small radius [Fig. 2(a)] configuration shows negligible hybridization between the $\mathbf{P}$ and $\mathbf{Q}$ bands, while some hybridization is observed if the sphere has a larger radius [Fig. 2(b)]. More information can be obtained by an eigenstate analysis. Let us focus on the quadrupole bands. In Fig. 2(a), taking the $\Gamma X$ direction as example, the lowest energy band is a $Q_{y z}$ quadrupole band with a small negative $v_{g}$. At higher energies the bands correspond to the twofolddegenerate states $Q_{x z}$ and $Q_{x y}$. The highest energy bands are the $Q_{y y}= \pm Q_{z z}$ bands with negative $v_{g}$. We note that $Q_{x x}$ is not a linear independent variable since $\operatorname{tr} \mathbf{Q}=0$. Three offdiagonal $\mathbf{Q}$ states are degenerate at the $\Gamma$ point and so are the two diagonal $\mathbf{Q}$ states, as required by symmetry. In Fig. 2(a), 
the agreement of black and green (red) dots indicates the independence of $\mathbf{P}$ and $\mathbf{Q}$ bands when the ratio $r_{s} / a$ is 0.26 . The hybridization between $\mathbf{P}$ and $\mathbf{Q}$ can be neglected. As the coupling between different angular-momentum channels is weak, the bands can be meaningfully assigned as dipole and quadrupole bands. As $r_{s} / a$ increases to 0.3 , longitudinal $\mathbf{P}$ and $Q_{y y}=Q_{z z}$ mode intersect at about $3.97 \mathrm{eV}$, which is shown in Fig. 2(b). The anticrossing effect can be observed and the original $\mathbf{P}$ and $\mathbf{Q}$ bands hybridize to form two new bands. In order to make the physics simple, we focus the following discussions on configurations which have a clean separation of $\mathbf{P}$ and $\mathbf{Q}$ derived bands.

\section{ANALYTICAL MODELING}

To obtain a better understanding of the band dispersions derived from the quadrupoles, we will set up a set of coupled quadruple equations. This is essentially the same strategy of setting up coupled dipole equations to study the dispersion of dipolar arrays. We start from the electric fields at point $\mathbf{r}$ generated by the quadrupole at the origin,

$$
\begin{aligned}
\mathbf{E}(\mathbf{r})= & -\frac{i}{6}\left[\left(f^{\prime}(r)-2 f(r) / r\right)(\mathbf{n} \cdot \mathbf{Q} \cdot \mathbf{n}) \mathbf{n}-\left(f^{\prime}(r)\right.\right. \\
& +f(r) / r) \mathbf{Q} \cdot \mathbf{n}],
\end{aligned}
$$

where $f(r)=k_{0}^{3} h_{2}^{(1)}\left(k_{0} r\right)$, and $\mathbf{n}=\mathbf{r} / r$ is an unit vector. We use the function $f(r)$ with argument $r$ instead of the spherical Hankel function $h_{2}^{(1)}\left(k_{0} r\right)$ with argument $k_{0} r$ for the sake of convenience. Since the quadrupoles respond linearly to the symmetric part of the gradient of the local electric field, we define a second-rank tensor field $\mathbf{F}$ as

$$
F_{\alpha \beta}=\operatorname{sym}(\nabla \mathbf{E})=\frac{1}{2}\left(\partial E_{\alpha} / \partial x_{\beta}+\partial E_{\beta} / \partial x_{\alpha}\right),
$$

where $\alpha$ and $\beta$ are indices of coordinates which run from 1 to 3. The gradient of field $\nabla \mathbf{E}$ can be calculated from Eq. (1). The diagonal and off-diagonal terms of $\mathbf{F}$ are, respectively,

$$
\begin{aligned}
F_{\alpha \alpha}= & -\frac{i}{6}\left[\left(f_{1} n_{\alpha}^{2}+f_{2}\right) \mathbf{n} \cdot \mathbf{Q} \cdot \mathbf{n}+f_{3} n_{\alpha}(\mathbf{Q} \cdot \mathbf{n})_{\alpha}+f_{4} \cdot Q_{\alpha \alpha}\right] \\
F_{\alpha \beta}= & -\frac{i}{6}\left[f_{1} n_{\alpha} n_{\beta} \mathbf{n} \cdot \mathbf{Q} \cdot \mathbf{n}+\frac{1}{2} f_{3}\left(n_{\beta}(\mathbf{Q} \cdot \mathbf{n})_{\alpha}+n_{\alpha}(\mathbf{Q} \cdot \mathbf{n})_{\beta}\right)\right. \\
& \left.+f_{4} Q_{\alpha \beta}\right],
\end{aligned}
$$

where $\nabla \cdot \mathbf{E}=0$ will be satisfied automatically since $\operatorname{tr} \mathbf{Q}=0$, and no Einstein summation convention is assumed. In Eq. (3), we define $f_{1}, f_{2}, f_{3}$, and $f_{4}$ as auxiliary functions which are linear combinations of $f(r), f^{\prime}(r)$, and $f^{\prime \prime}(r)$,

$$
\begin{gathered}
f_{1}=f^{\prime \prime}(r)-5 f^{\prime}(r) / r+8 f(r) / r^{2}, \quad f_{2}=f^{\prime}(r) / r-2 f(r) / r^{2}, \\
f_{3}=-f^{\prime \prime}(r)+2 f^{\prime}(r) / r-2 f(r) / r^{2}, \quad f_{4}=-f^{\prime}(r) / r-f(r) / r^{2} .
\end{gathered}
$$

From Eq. (3) the Green's function $\mathbf{G}^{Q}\left(\mathbf{r}-\mathbf{r}^{\prime}\right)$ for the second-rank tensor field $\mathbf{F}$ can be defined, which satisfies

$$
\mathbf{F}(\mathbf{r})=\mathbf{G}^{Q}\left(\mathbf{r}-\mathbf{r}^{\prime}\right) \mathbf{Q}\left(\mathbf{r}^{\prime}\right) .
$$

To deal with the second-rank tensor in a compact form, we can rewrite $\mathbf{Q}$ and $\mathbf{F}$ as vectors: $\mathbf{Q}$ $=\left(Q_{22}, Q_{33}, Q_{12}, Q_{13}, Q_{23}\right)^{T}$ and $\mathbf{F}=\left(F_{22}, F_{33}, F_{12}, F_{13}, F_{23}\right)^{T}$. In this vector notation, the Green's function $\mathbf{G}^{Q}\left(\mathbf{r}-\mathbf{r}^{\prime}\right)$ is represented by a $5 \times 5$ matrix.

Once the local tensor field $\mathbf{F}$ is given, the linear response is simply $\mathbf{Q}=\alpha_{Q} \mathbf{F}$. Considering a quadrupole at position $\mathbf{r}_{i}$ in the lattice, the corresponding local tensor field $\mathbf{F}$ is generated by all the other quadrupoles except itself, namely,

$$
\mathbf{Q}\left(\mathbf{r}_{i}\right)=\alpha_{Q} \mathbf{F}\left(\mathbf{r}_{i}\right)=\alpha_{Q} \sum_{\mathbf{r}_{j} \neq \mathbf{r}_{i}} \mathbf{G}^{Q}\left(\mathbf{r}_{i}-\mathbf{r}_{j}\right) \mathbf{Q}\left(\mathbf{r}_{j}\right) .
$$

The Green's function $\mathbf{G}^{Q}\left(\mathbf{r}-\mathbf{r}^{\prime}\right)$ can be obtained from Eq. (3). With the Bloch condition imposed on Eq. (5), we will reproduce the rigorous results given by three-dimensional MST with angular momentum $l=2$ only. Since $\mu=1$ for the plasmonic spheres here, the contribution from magnetic quadrupole is not considered here. We see from Fig. 2(a) that for plasmonic lattices in which the $r_{s} / a$ ratio is smaller than 0.26 , the bands for $l=1$ and $l=2$ has no observable hybridization, and the two angular-momentum channels are indeed separable. We can thus discuss "quadruple bands" and "dipole bands" separately. For the quadrupole bands, the dispersion is analogous to $d$ bands in transition metals. ${ }^{11}$ For high angular-momentum states, the coupling is typically short ranged. The short-range interaction is dominated by the near zone field in electrodynamics, or in other words, the quasistatic limit ${ }^{19,20}$ of EM fields. We will show that the quadrupole bands obtained within the framework of the quasistatic approximation (QSA) agree very well with the full electrodynamic results.

In the quasistatic limit, we take the light speed $c$ to infinity and the electric field $\mathbf{E}$ does not depend on $k_{0}$. In that limit, only the terms containing the lowest power of $r$ survive and the auxiliary functions $f_{i}$ are reduced to the simple forms

$$
f_{1} \sim-105 i / r^{5}, \quad f_{2} \sim 15 i / r^{5}, \quad f_{3} \sim 60 i / r^{5}, \quad f_{4} \sim-6 i / r^{5} .
$$

In the quasistatic limit, all slowly convergent powers of $r$ in $\nabla \mathbf{E}$ drop out, and we need to consider only the $r^{-5}$ terms, which simplify the lattice sum in Eq. (5) considerably. Special summation techniques developed to accelerate convergence such as Ewald's sum is unnecessary in the QSA here. Equations (3) and (6) give us the quadrupole's Green's function in QSA with a simple $5 \times 5$ matrix form. As is known from the band-structure analysis above, the eigenstates of the two quadrupole negative bands contain only the diagonal elements $\left\{Q_{y y}, Q_{z z}\right\}$ of the $\mathbf{Q}$ tensor. The subspace spanned by their eigenvectors is orthogonal to that formed by the nondiagonal elements. The bands of these $\left\{Q_{y y}, Q_{z z}\right\}$ states are of special interest since they have intrinsic negative group velocities. $\mathbf{F}$ and $\mathbf{Q}$ are reduced to two-dimensional vectors when we focus on the subspace spanned by $Q_{y y}$ and $Q_{z z}$ that give two quadrupole bands with negative group velocity. We define two-dimensional vectors $Q^{\prime}=\left(Q_{y y}, Q_{z z}\right)$, $\boldsymbol{F}^{\prime}=\left(F_{y y}, F_{z z}\right)$, and the $2 \times 2$ matrix form of QSA Green's function becomes 


$$
\mathbf{G}^{\prime}(\mathbf{r})=\frac{1}{6 r^{5}}\left[\begin{array}{ll}
105 n_{y}^{2}\left(n_{x}^{2}-n_{y}^{2}\right)+75 n_{y}^{2}-15 n_{x}^{2}-6 & 15\left(n_{x}^{2}-n_{z}^{2}\right)\left(7 n_{y}^{2}-1\right) \\
15\left(n_{x}^{2}-n_{y}^{2}\right)\left(7 n_{z}^{2}-1\right) & 105 n_{z}^{2}\left(n_{x}^{2}-n_{z}^{2}\right)+75 n_{z}^{2}-15 n_{x}^{2}-6
\end{array}\right],
$$

where $\mathbf{n}$ is again the unit vector in the direction of $\mathbf{r}$.

To find the Bloch states, the lattice Green's function is transformed to the Fourier space: $\mathbf{G}^{\prime}(\mathbf{k})=\sum_{r_{i} \neq 0} \mathbf{G}^{\prime}\left(\mathbf{r}_{i}\right) e^{i \mathbf{k} \cdot \mathbf{r}_{i}}$. Equation (5) becomes a secular equation of the form $\operatorname{det}\left[\alpha_{Q}^{-1} \mathbf{I}-\mathbf{G}^{\prime}(\mathbf{k})\right]=0$, where $\alpha_{Q}^{-1}$ and $\mathbf{G}^{\prime}(\mathbf{k})$ contain the material and geometric information, respectively. The dyadic Green's function in Eq. (7) now has a simple structure since the only dependence on the lattice constant is the prefactor $r^{-5}$. We can evaluate the lattice sum in a mesh of integer points $(i, j, k)$ which are independent of the lattice constant to solve the secular equation. In Fig. 3(a), we can see that the QSA dispersion relations which are shown by light gray (green) dots for the states spanned by $Q_{y y}$ and $Q_{z z}$ agree well with the MST results (black dots). Since the QSA gives such good agreement for the quadrupole bands, we can now discuss the physics using the QSA picture to give a more straightforward interpretation and we can obtain analytic results near the zone center. At the $\Gamma$ point the lattice Green's function is found to be $\mathbf{G}^{\prime}$ $=-a^{-5} \sigma \cdot \mathbf{I}$, where $\sigma$ is a lattice sum,

$$
\begin{aligned}
\sigma= & \frac{1}{6\left(i^{2}+j^{2}+k^{2}\right)^{5 / 2}} \sum_{(i, j, k) \neq 0} 105 j^{2}\left(i^{2}-j^{2}\right) /\left(i^{2}+j^{2}+k^{2}\right)^{2} \\
& +\left(75 j^{2}-15 i^{2}\right) /\left(i^{2}+j^{2}+k^{2}\right)-6 .
\end{aligned}
$$

$\sigma$ can be easily summed to give a value of 18.65 . This is a constant that is specific to the geometry of the system. The
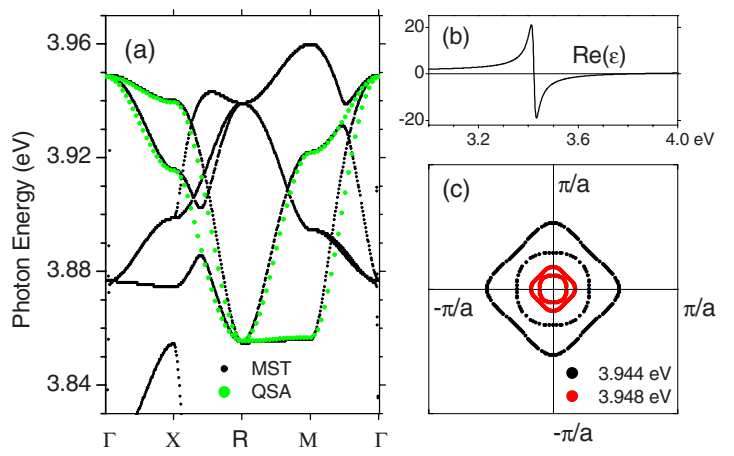

FIG. 3. (Color online) (a) The dispersion of negative group velocity bands calculated under QSA which is shown by light gray (green) dots compared with the rigorous MST results (black dots). The lattice constant $a$ is $20 \mathrm{~nm}$ and radius $r_{s}$ is $5.2 \mathrm{~nm}$. (b) The effective permittivity $\varepsilon_{e f f}\left(\mu_{e f f}=1\right.$, not shown) calculated by CPA where we use the electron collision frequency $\gamma=0.02 \mathrm{eV}$ in the Drude model. Quadrupole bands near $3.9 \mathrm{eV}$ are beyond the description of CPA. (c) Equifrequency contours of the two negative bands. The frequencies are 3.948 and $3.944 \mathrm{eV}$ for dark gray (red) and black dots, respectively. The band with smaller negative slope has square shape even near the Brillouin-zone center, while the one with larger negative slope is more isotropic. quadrupole polarizabilty in QSA has a simple form $\alpha_{Q}^{-1} \rightarrow\left(1-5 \omega^{2} / 2 \omega_{p}^{2}\right) r_{s}^{-5}$ if the Drude-type permittivity is applied. Combining the dynamical and geometric parts in the secular equation, we get the QSA frequency of eigenstate at the $\Gamma$ point,

$$
\omega_{0}=\omega_{p} \sqrt{\frac{2}{5}\left(1+\sigma\left(r_{s} / a\right)^{5}\right)} .
$$

The term of $\left(r_{s} / a\right)^{5}$ can be regarded as the geometric correction of the single sphere quadrupole resonance $\omega_{l=2}$ $=\sqrt{\frac{2}{5}} \omega_{p}$. This semianalytic result indicates the symmetry required degeneracy of two eigenstates $Q_{y y}= \pm Q_{z z}$ at the $\Gamma$ point for the simple-cubic lattice, which is manifested in the numerical calculations, such as the example shown in Fig. 1 with $r_{s} / a=0.26$. The result given by formula (8): $\omega_{0}$ $=3.95 \mathrm{eV}$ is the same as the numerical result from MST up to two decimal places when $a=20 \mathrm{~nm}$.

To show that the group velocity is intrinsically negative, we find the group velocity and the second derivative of the photon dispersion near the $\Gamma$ point. We do the first and second derivatives on the secular equation. The first derivative $\nabla_{\mathbf{k}} \mathbf{G}^{\prime}$ is found to be exactly zero, thus the group velocity is $\partial \omega / \partial k=0$ as expected. The second derivative of the dispersion at the zone center is found to be direction dependent. Taking $\Gamma \mathrm{X}$ and $\Gamma \mathrm{M}$ direction as examples, we obtain

and

$$
\partial^{2} \mathbf{G}^{\prime} /\left.\partial k^{2}\right|_{\Gamma \mathrm{X}}=a^{-3}\left[\begin{array}{ll}
4.71 & 2.86 \\
2.86 & 4.71
\end{array}\right],
$$

$$
\partial^{2} \mathbf{G}^{\prime} /\left.\partial k^{2}\right|_{\Gamma \mathrm{M}}=a^{-3}\left[\begin{array}{cc}
4.71 & -1.43 \\
-1.43 & 4.71
\end{array}\right] .
$$

The second derivative of secular equation finally gives us the results: $\partial^{2} \omega /\left.\partial k^{2}\right|_{\Gamma \mathrm{X}}=(-4.71 \pm 2.86) m_{c}^{-1}$ and $\partial^{2} \omega /\left.\partial k^{2}\right|_{\Gamma \mathrm{M}}$ $=(-4.71 \pm 1.43) m_{c}^{-1}$, where $m_{c}=5 \omega_{0} a^{3} / \omega_{p}^{2} r_{s}^{5}$ is a system parameter. This shows that the dispersion is necessarily anisotropic, even for a system with cubic symmetry. The inverse of second derivative of the plasmonic dispersion corresponds to the effective mass in electronics. The band with a larger absolute value of the second derivative has a more negative slope and a lower frequency. Also it is clear that the contrast $m_{\Gamma \mathrm{X}} / m_{\Gamma \mathrm{M}}$ is closer to 1 for the lower frequency band. The equifrequency contours of these negative bands cannot be simply represented by ellipsoids. In photonic crystals, similar anisotropy near the $\Gamma$ point have also been be observed, ${ }^{21}$ which is essentially introduced by multipoles with $l \geq 2$. Figure 3(c) shows the equifrequency contours of the two negative bands. The lower frequency band with larger negative slope has more circular shape as expected, while the higher frequency band is distinctly anisotropic. By checking 
the eigenstates, the lower frequency band corresponds to the eigenstates $Q_{y y}=Q_{z z}$ in the $\Gamma \mathrm{X}$ direction and $Q_{y y}=-Q_{z z}$ in the $\Gamma \mathrm{M}$ direction, respectively.

The above results show that the bands derived from $Q_{y y}$ and $Q_{z z}$ are intrinsically negative in group velocity in a simple-cubic array. We should emphasize here that the negative group velocity bands differ fundamentally from that given by other mechanisms. For example, we can obtain negative group velocity in a lattice by Bragg scattering which is typically the case in dielectric photonic crystals. In those cases, the frequency of negative dispersion bands must be scale dependent, which means that the frequency scales inversely with the lattice parameter. However, for plasmonic lattices, the dispersions are nearly scale independent, as shown in Fig. 1. Another way to obtain negative dispersion is through "double negativity" by the coupling of electric and magnetic dipoles, as proposed by Veselago. ${ }^{1}$ In that case, the electric-dipole and magnetic-dipole resonances are strong enough that they can each give, respectively, negative $\varepsilon$ and $\mu$. Although each resonance $\mathbf{P}$ or $\mathbf{M}$ on its own gives a band gap, when they couple together in the same frequency, a pass band with a negative group velocity emerges. The intrinsic negative group velocity bands due to the electric quadrupoles are different since the mechanism is not the coupling of electric and magnetic resonances, but the hopping of one type of electric resonance (quadrupole) from one site to another. In this aspect, the physics is rather similar to the tight-binding picture of electrons. We note in particular that it is not meaningful to extract effective permittivity or permeability from such a system (assuming that we use simple homogenization theories that give $\mathbf{k}$-independent permittivity and permeability). If we insist on applying standard inversion procedures (say by using the amplitude and phase of transmission and reflection to extract some form of negative $\varepsilon$ and $\mu,{ }^{22}$ field averaging method, ${ }^{23}$ or other effective-medium theory such as coherent-potential approximation (CPA), ${ }^{24}$ the extracted constitutive parameters carry no physical meaning. In Fig. 3(b), we calculate the effective parameters $\varepsilon_{\text {eff }}\left(\mu_{\text {eff }}=1\right.$ from the extraction procedure, not shown) using CPA, where even no propagating modes exist near the quadrupole resonance $3.9 \mathrm{eV}$ as $\varepsilon_{e f f}$ is negative.

\section{PLASMONIC CLUSTERS}

The underlying physics can also be understood from the constitutive relation of macroscopic Maxwell equations: $D_{\alpha}$ $=E_{\alpha}+4 \pi P_{\alpha}-4 \pi \Sigma_{\beta} \partial_{\beta} Q_{\alpha \beta}$ (see Ref. 25, Chap. 6). Near the quadrupole resonance, the quadrupole terms cannot be neglected, and therefore the electric permittivity cannot be determined by the electric susceptibility $\chi_{e}$ alone. ${ }^{26}$ The coupling between magnetic-dipole and electric-quadrupole resonances is emphasized in metamaterial design. In the optical frequency range, the electric-quadrupole terms are in general of the same order of magnitude as the magneticdipole terms, and it is probably prudent to consider the effect of electric quadrupole whenever magnetic-dipoles resonances are strong, ${ }^{10}$ unless one can argue that the electricquadrupole effect is zero due to symmetry or negligible with respect to the magnetic dipole for some particular reason.
This point can be illustrated by a prototypical plasmonic lattice system in which the building block comprises of a cluster of metallic nanoparticles. Here we consider only lowfrequency excitations, and no high multipole $(l \geq 2)$ for a single nanoparticle can be excited. In this case every nanoparticle can be treated as a dipole, and we can use coupled dipole equations to describe the system. ${ }^{15}$ Let us first consider a finite system with $N$ point dipoles $\mathbf{p}_{i}$ located at $\mathbf{r}_{i}$, $\{i=1, N\}$. As the dimensions are small compared to the wavelength, i.e., $k_{0} r_{i} \ll 1$, we can do a multipole expansion following standard procedures (see Ref. 25, Chap. 9). The time-dependent current contributed by each dipole is $\mathbf{J}_{i}$ $=\partial \mathbf{p}_{i} / \partial t \delta\left(\mathbf{r}-\mathbf{r}_{i}\right)=-i \omega \mathbf{p}_{i} \delta\left(\mathbf{r}-\mathbf{r}_{i}\right)$. By substituting this current to the definition of electric, magnetic dipole, and electric quadrupole, and applying the continuity equation $\partial \rho / \partial t$ $+\nabla \cdot \mathbf{J}=0$, it is straightforward to find $\mathbf{P}=\int \mathbf{x} \rho(\mathbf{x}) d^{3} x$ $=-\frac{1}{i \omega} \int \mathbf{J} d^{3} x, \quad \mathbf{M}=\frac{1}{2 c} \int \mathbf{x} \times \mathbf{J} d^{3} x, \quad$ and $\quad \overline{\mathbf{Q}}=3 \int \mathbf{x} \mathbf{x} \rho(\mathbf{x}) d^{3} x$ $=-\frac{3}{i \omega} \int(\mathbf{x} \mathbf{J}+\mathbf{J} \mathbf{x}) d^{3} x$. (Here we use the $\overline{\mathbf{Q}}$ for convenience, the proper definition of quadrupole should be modified to be traceless: $\mathbf{Q}=\mathbf{Q}-\frac{1}{3} \operatorname{tr} \overline{\mathbf{Q}}$.) The corresponding total electric, magnetic dipole, and quadrupole moments are $\mathbf{P}=\Sigma \mathbf{p}_{i}, \mathbf{M}$ $=-\frac{i k_{0}}{2} \sum \mathbf{r}_{i} \times \mathbf{p}_{i}$, and $\mathbf{Q}=3 \sum\left(\mathbf{r}_{i} \mathbf{p}_{i}+\mathbf{p}_{i} \mathbf{r}_{i}\right)$ respectively. It can be demonstrated by the far-field expansion that the EM fields in the far field due to the set of $N$ dipoles is indeed equivalent to a radiator with such values of $\mathbf{P}, \mathbf{M}$, and $\mathbf{Q}$ as defined by $\mathbf{P}=\sum \mathbf{p}_{i}, \mathbf{M}=-\frac{i k_{0}}{2} \sum \mathbf{r}_{i} \times \mathbf{p}_{i}$, and $\mathbf{Q}=3 \sum\left(\mathbf{r}_{i} \mathbf{p}_{i}+\mathbf{p}_{i} \mathbf{r}_{i}\right)$ to the zeroth and first order. We will also consider in the Appendix the more general case where the size of the plasmonic cluster does not have to be small compared to the wavelength. If the particles are small and close together, the $\mathbf{P}$ and $\mathbf{Q}$ formulas are proved to be accurate numerically, while the formula for $\mathbf{M}$ has a very simple analytical form as shown in the Appendix. It is well known that a current loop yields a nonzero magnetic-dipole moment, and a vanishing quadrupole moment. However, the quadrupole moment contributed by the current generated by discrete electric dipoles $\mathbf{p}_{i}$ is usually not zero, and has the same order $L \cdot P$ as the magnetic dipole, where $L$ is the characteristic linear size of the cluster, and $P$ is the magnitude of electric dipoles. To find a "pure magnetic dipole" $(\mathbf{M} \neq 0, \mathbf{Q}=0)$ or a "pure quadrupole" $(\mathbf{Q} \neq 0, \mathbf{M}$ $=0)$ in a frequency range, some particular symmetry is required to guarantee one of them to be zero. The behavior of $\mathbf{P}, \mathbf{M}$, and $\mathbf{Q}$ in a plasmonic cluster could strongly deviate from a single particle since their resonant frequencies rely on the special geometry of the configuration. A useful eigenmode decomposition method has been adopted to investigate dipole clusters ${ }^{15,27}$ or one dimensional chain. ${ }^{14}$ We choose the configuration of the cluster to be an octahedron, which is shown in the inset of Fig. 4. The radius of sphere is $r_{s}$ $=5.6 \mathrm{~nm}$, and the spacing between opposite corners is $R$ $=24 \mathrm{~nm}$. The octahedron structure has symmetry group $O_{h}$ which may support negative group velocity in $x, y$, and $z$ directions simultaneously.

With $\mathbf{P}, \mathbf{M}$, and $\mathbf{Q}$ defined above, we can classify the eigenstates. Given a particular frequency, we can solve for the eigenvectors that give the values of $\mathbf{p}_{i}$ on each site, and the $\mathbf{P}, \mathbf{M}$, and $\mathbf{Q}$ formulas give us the corresponding dipole and quadrupole moments. The octahedral system has many types of multipole resonances. Figure 4(a) shows four differ- 


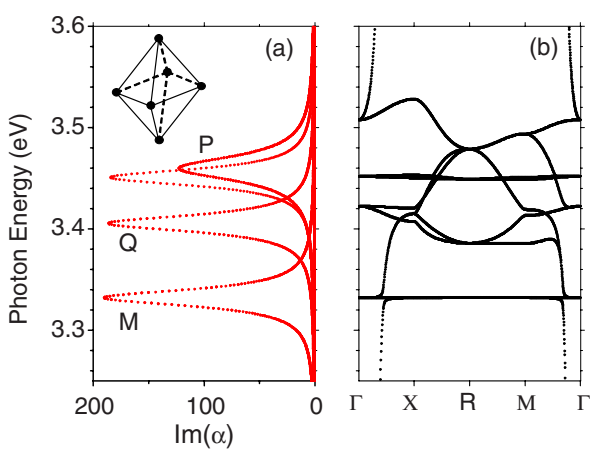

FIG. 4. (Color online) (a) The imaginary part of the linear response function for an octahedron composed of six sphere with sphere radius $r_{s}=5.6 \mathrm{~nm}$, and the spacing between opposite corners is $R=24 \mathrm{~nm}$. The collective resonance with the lowest frequency at $3.33 \mathrm{eV}$ is a magnetic dipole. The resonance at $3.41 \mathrm{eV}$ is a quadrupole. And the ones near $3.45 \mathrm{eV}$ are an electric dipole accompanied with another higher multipole. (b) Band structure of a simplecubic plasmonic lattice with lattice constant $a=60 \mathrm{~nm}$ when the octahedron is used to be the building block in a unit cell. The negative group velocity band can be observed near quadrupole resonance frequency $3.4 \mathrm{eV}$.

ent eigenstates below $3.6 \mathrm{eV}$. The lowest one at $3.33 \mathrm{eV}$ is a magnetic-dipole eigenstate, which means only the total magnetic moment $\mathbf{M} \neq 0$, and meanwhile $\mathbf{P}, \mathbf{Q}=0$. The next one at $3.41 \mathrm{eV}$ is a quadrupole state, and the one at $3.45 \mathrm{eV}$ is found to be electric dipole accompanied by another higher multipole with $l \geq 3$. The interaction among dipoles in the octahedron can produce $\mathbf{M}$ and $\mathbf{Q}$ resonances. We note that in this particular structure with the symmetry group $O_{h}$, the "pure magnetic dipole" and "pure quadrupole" states can be found and will not mix with each other. However, the $\mathbf{M}$ and $\mathbf{Q}$ resonances are rather close in frequency, which reinforces the notion that for most plasmonic structures (not just the octahedron) the $\mathbf{M}$ and $\mathbf{Q}$ resonances come together in frequency. Although we neglect all the dynamic multipoles $l$ $\geq 2$ in such low frequencies for a single sphere, multipole resonances can be generated by the geometry of the configuration.

If this plasmonic cluster with octahedron geometry is used as the building block of three-dimensional lattices, the corresponding band structure can be classified by the $\mathbf{P}, \mathbf{M}$, and $\mathbf{Q}$ eigenstate defined above. In Fig. 4(b), the band structure of a simple-cubic lattice with lattice constant $a=60 \mathrm{~nm}$ is shown. A negative group velocity band can be observed for quadrupole state near $3.4 \mathrm{eV}$. Although similar quadrupole bands with negative group velocity have been found for single sphere near $3.9 \mathrm{eV}$ in Sec. II, the more complex building block can lower the resonance frequency considerably. Many types of building blocks, such as nanorod pairs, "fishnet" structures, ${ }^{5}$ are introduced to realize negative refraction metamaterial in optical frequencies. The negative permeability $\mu<0$ is usually considered to be associated with a magnetic-dipole resonance. Actually for a far-field observer, the contribution from electric quadrupoles might be comparable to that from magnetic dipoles, which is demonstrated by simulations. ${ }^{10}$ Since in optical frequencies, $\varepsilon$ and $\mu$ are not uniquely defined, ${ }^{8,9}$ one can describe all electromagnetic responses by dielectric function $\varepsilon(\omega, \mathbf{k})$ with both time and spatial dispersion and set $\mu$ as 1 . Or equivalently, especially in the case of metamaterials, people usually prefer a description of $\mathbf{k}$-independent effective $\varepsilon(\omega)$ and $\mu(\omega)$ to achieve the "double negativity." Our calculation shows the quadrupole itself can render the negative refraction possible even in the absence of electric- and magnetic-dipole resonance, where the effective-medium description by $\varepsilon(\omega)$ and $\mu(\omega)$ might not carry useful meaning.

\section{CONCLUSION}

In summary, we analytically proved that two branches of quadrupole bands in simple-cubic plasmonic lattices consisting of metallic spheres with very small lattice constants have intrinsic negative group velocities. The mechanism responsible for this new type of negative dispersion is analogous to that for $d$-state electrons in transition metals and its quadrupole nature goes beyond conventional isotropic effectivemedium theory. Since the short-range interaction dominates, the QSA is found to be very accurate for quadrupoles as the filling ratio is small. In the typical plasmonic system composed of metallic nanoparticle clusters, the quadrupoles play a significant role as their resonance can be adjusted to lower optical frequencies, and the coupling strength to external EM waves are in the same order of magnitude as $\mathbf{M}$. This can be very important in the design of metamaterials.

\section{ACKNOWLEDGMENTS}

This work was supported by the Central Allocation Grant from the Hong Kong RGC through HKUST3/06C. Computation resources were supported by the Shun Hing Education and Charity Fund. We thank Xianyu Ao, Jack Ng, and Junjun Xiao for helpful discussions.

\section{APPENDIX: EFFECTIVE DIPOLE AND QUADRUPOLE MOMENTS FROM RIGOROUS EXPANSION METHOD}

We use the same notations for the vector spherical harmonics as those defined in Ref. 28. And $j_{l}\left(k_{0} r\right)$ and $h_{l}\left(k_{0} r\right)$ are, respectively, spherical Bessel functions and spherical Hankel functions of the first kind. As shown in Ref. 28, the regular and irregular vector spherical solid harmonics can be expanded in terms of a "structure constant."

$$
\left|\mathbf{H}_{l m \sigma}(\mathbf{r}-\mathbf{R})\right\rangle=\sum_{l^{\prime} m^{\prime} \sigma^{\prime}} G_{l m \sigma ; l^{\prime} m^{\prime} \sigma^{\prime}}(\mathbf{R})\left|\mathbf{H}_{l^{\prime} m^{\prime} \sigma^{\prime}}(\mathbf{r})\right\rangle
$$

where the general form of the structure constants $G_{l m \sigma: l^{\prime} m^{\prime} \sigma^{\prime}}(\mathbf{R})$ can be found in Ref. 28 and references therein. Applying the relations above, we can "shift" the electric dipole $\mathbf{P}$ at position $\mathbf{R}$ to electric dipole $\mathbf{P}^{\prime}$, magnetic dipole $\mathbf{M}^{\prime}$, quadrupole $\mathbf{Q}^{\prime}$ and higher multipoles at the origin. The structure constants can be simplified by the dipole approximation (namely, only electric dipole $\mathbf{P}$ at position $\mathbf{R}$ ), 


$$
G_{1 m E ; l^{\prime} m^{\prime} \sigma^{\prime}}(\mathbf{R})=\left\{\begin{array}{c}
\sum_{\mu} C(1,1,1 ; m-\mu, \mu) g_{1, m-\mu ; l^{\prime}, m^{\prime}-\mu}(\mathbf{R}) C\left(l^{\prime}, 1, l^{\prime} ; m^{\prime}-\mu, \mu\right), \\
\text { when } \quad \sigma^{\prime}=E \\
-\sqrt{\frac{2 l^{\prime}+1}{l^{\prime}+1} \sum_{\mu} C(1,1,1 ; m-\mu, \mu) g_{l, m-\mu ; l^{\prime}-1, m^{\prime}-\mu}(\mathbf{R}) C\left(l^{\prime}-1, l^{\prime}, 1 ; m^{\prime}-\mu, \mu\right)} \\
\text { when } \quad \sigma^{\prime}=M .
\end{array}\right.
$$

Here $g_{l, m ; l^{\prime}, m^{\prime}}(\mathbf{R})$ are structure factors for the scalar waves, $C$ are the Clebsch-Gordon coefficients between the angular momentum 1 and $l$ which combine the vector nature and spatial dependence of EM waves. The scalar structure factors are given by

$$
g_{l, m ; l^{\prime}, m^{\prime}}(\mathbf{R})=4 \pi \sum_{l^{\prime \prime} m^{\prime \prime}} l^{l^{\prime}+l^{\prime \prime}-l} C_{l m ; l^{\prime} m^{\prime} ; l^{\prime \prime} m^{\prime \prime} j_{l^{\prime \prime}}}\left(k_{0} R\right) Y_{l^{\prime \prime} m^{\prime \prime}}(-\hat{\mathbf{R}}) .
$$

$C_{l m ; l^{\prime} m^{\prime} ; l^{\prime \prime} m^{\prime \prime}}$ are the Gaunt coefficients which determine the overlap coefficients among three spherical harmonics,

$$
C_{l m ; l^{\prime} m^{\prime} ; l^{\prime \prime} m^{\prime \prime}}=\int d \Omega_{k} Y_{l^{\prime} m^{\prime}}^{*}(\hat{k}) Y_{l^{\prime \prime} m^{\prime \prime}}^{*}(\hat{k}) Y_{l m}(\hat{k}) .
$$

The electric field for the dipole $\mathbf{P}$ at position $\mathbf{R}$ can be expanded as vector spherical harmonics centered at the origin,

$$
\mathbf{E}(\mathbf{r})=\sum_{l m \sigma} b_{l m}^{\sigma}\left|\mathbf{H}_{l m \sigma}(\mathbf{r})\right\rangle .
$$

After the rigorous expansion, one should find the relation between the coefficients of multipoles $l=1,2$ to the components of $\mathbf{P}^{\prime}, \mathbf{M}^{\prime}$, and $\mathbf{Q}^{\prime}$ centered at the origin by comparing the electric field with the corresponding electric fields radiated by $\mathbf{P}^{\prime}, \mathbf{M}^{\prime}$, and $\mathbf{Q}^{\prime}$. The matrices $\mathbf{T}_{p b}, \mathbf{T}_{m b}$, and $\mathbf{T}_{Q b}$ can be introduced which satisfy $\mathbf{P}^{\prime}=\mathbf{T}_{p b} \mathbf{b}^{(E)}, \mathbf{M}^{\prime}=\mathbf{T}_{m b} \mathbf{b}^{(M)}$, and $\mathbf{Q}^{\prime}=\mathbf{T}_{Q b} \mathbf{b}^{(Q)}$. Here, $\mathbf{b}^{(E)}, \mathbf{b}^{(M)}$, and $\mathbf{b}^{(Q)}$ are the coefficients defined by $\mathbf{b}^{(E)}=\left(b_{1,-1}^{(E)}, b_{1,0}^{(E)}, b_{1,1}^{(E)}\right), \quad \mathbf{b}^{(M)}=\left(b_{1,-1}^{(M)}, b_{1,0}^{(M)}, b_{1,1}^{(M)}\right)$, and $\mathbf{b}^{(Q)}=\left(b_{2,-2}^{(E)}, \cdots, b_{2,2}^{(E)}\right)$. The matrices $\mathbf{T}_{p b}$ and $\mathbf{T}_{m b}$ are found to be

$$
\mathbf{T}_{p b}=\mathbf{T}_{m b}=\frac{1}{i k_{0}^{3}} \sqrt{\frac{3}{16 \pi}}\left[\begin{array}{ccc}
1 & 0 & -1 \\
-i & 0 & i \\
0 & \sqrt{2} & 0
\end{array}\right],
$$

while $\mathbf{T}_{Q b}$ is a $5 \times 5$ matrix (see Ref. 25 , Chap. 4) which we are not going to show here. Now we can use $\mathbf{T}_{p b}^{-1}$ to transform electric dipole $\mathbf{P}(\mathbf{r}=\mathbf{R})$ to $\mathbf{b}^{(E)}(\mathbf{r}=\mathbf{R})$, then we can find $\mathbf{b}^{(E, M, Q)}(\mathbf{r}=0)$ at the origin using the known structure constants $G_{1 m E: l^{\prime} m^{\prime} \sigma^{\prime}}(\mathbf{R})$. Finally we transform $\mathbf{b}^{(E, M, Q)}(\mathbf{r}=0)$ back to $\mathbf{P}^{\prime}, \mathbf{M}^{\prime}$, and $\mathbf{Q}^{\prime}$ by $\mathbf{T}_{p b}, \mathbf{T}_{m b}$, and $\mathbf{T}_{Q b}$, respectively. Numerically we have proved that if the ratio $R / \lambda$ is smaller than 0.1, the total moments $\mathbf{P}^{\prime}, \mathbf{M}^{\prime}$, and $\mathbf{Q}^{\prime}$ calculated by the far-field expansion that shown in Sec. IV are correct within the error 10\%. Hence, these formulas in Sec. IV are good for "small objects," and by small, we mean small compared with the wavelength.

However, the total magnetic moment $\mathbf{M}^{\prime}$ have a quite simple form since only the $l^{\prime}=m^{\prime}=0$ terms contribute to the scalar structure factors $g_{l, m ; l^{\prime}, m^{\prime}}(\mathbf{R})$ given by (A2). In this case the Gaunt coefficients $C_{l m: l^{\prime} m^{\prime} ; l^{\prime \prime} m^{\prime \prime}}$ can be evaluated as

$$
C_{l m ; 00 ; l^{\prime \prime} m^{\prime \prime}}=\int d \Omega_{k} Y_{00}^{*}(\hat{k}) Y_{l^{\prime \prime} m^{\prime \prime}}^{*}(\hat{k}) Y_{l m}(\hat{k})=\frac{1}{\sqrt{4 \pi}} \delta_{l l^{\prime \prime}} \delta_{m m^{\prime \prime}},
$$

and we have $g_{l m ; 00}(\mathbf{R})=\sqrt{4 \pi} j_{l}\left(k_{0} R\right) Y_{l m}(-\hat{\mathbf{R}})$. The structure constants are

$$
\begin{aligned}
& G_{1 m E ; 1 m^{\prime} M}(\mathbf{R}) \\
& \quad=-\sqrt{6 \pi} C\left(1,1,1 ; m-m^{\prime}, m^{\prime}\right) j_{1}\left(k_{0} R\right) Y_{1 m}(-\hat{\mathbf{R}}),
\end{aligned}
$$

where $C\left(0,1,1 ; 0, m^{\prime}\right)=1$ is used. It can be rewritten in a matrix form

$$
\mathbf{G}(\mathbf{R})=\sqrt{3 \pi} j_{1}\left(k_{0} R\right)\left[\begin{array}{ccc}
Y_{10}(\hat{\mathbf{R}}) & -Y_{1,-1}(\hat{\mathbf{R}}) & 0 \\
Y_{11}(\hat{\mathbf{R}}) & 0 & -Y_{1,-1}(\hat{\mathbf{R}}) \\
0 & Y_{11}(\hat{\mathbf{R}}) & -Y_{10}(\hat{\mathbf{R}})
\end{array}\right],
$$

where we use that $Y_{1 m}(-\hat{\mathbf{R}})=-Y_{1 m}(\hat{\mathbf{R}})$. The matrix which "shifts" electric dipole $\mathbf{P}$ at position $\mathbf{R}$ to magnetic dipole $\mathbf{M}^{\prime}$ at $\mathbf{r}=0$ is $\mathbf{T}_{m b} \mathbf{G}^{T}(\mathbf{R}) \mathbf{T}_{p b}^{-1}$. After some algebra, we find that

$$
\begin{aligned}
\mathbf{T}_{m b} \mathbf{G}^{T}(\mathbf{R}) \mathbf{T}_{p b}^{-1} & =\frac{3 i}{2} j_{1}\left(k_{0} R\right)\left[\begin{array}{ccc}
0 & \cos \theta & -\sin \theta \sin \phi \\
-\cos \theta & 0 & \sin \theta \cos \phi \\
\sin \theta \sin \phi & -\sin \theta \cos \phi & 0
\end{array}\right] .
\end{aligned}
$$

The matrix in Eq. (A7) may look complicated, but it is exactly the definition of vector product,

$$
\mathbf{M}^{\prime}=-\frac{3 i}{2} \frac{j_{1}\left(k_{0} R\right)}{R} \mathbf{R} \times \mathbf{P} .
$$

In the long-wavelength limit $k_{0} R \ll 1, j_{1}(x) \rightarrow \frac{1}{3} k_{0} R$, Eq. (A8) becomes $\mathbf{M}^{\prime}=-\frac{i k_{0}}{2} \mathbf{R} \times \mathbf{P}$, which is consistent with the result from the far-field expansion in the text. 
${ }^{1}$ V. G. Veselago, Sov. Phys. Usp. 10, 509 (1968).

${ }^{2}$ J. B. Pendry, Phys. Rev. Lett. 85, 3966 (2000).

${ }^{3}$ R. A. Shelby, D. R. Smith, and S. Schultz, Science 292, 77 (2001).

${ }^{4}$ V. M. Shalaev, W. S. Cai, U. K. Chettiar, H. K. Yuan, A. K. Sarychev, V. P. Drachev, and A. V. Kildishev, Opt. Lett. 30, 3356 (2005).

${ }^{5}$ S. Zhang, W. Fan, K. J. Malloy, S. R. J. Brueck, N. C. Panoiu, and R. M. Osgood, Opt. Express 13, 4922 (2005); G. Dolling, C. Enkrich, M. Wegener, C. M. Soukoulis, and S. Linden, Science 312, 892 (2006); J. Valentine, S. Zhang, T. Zentgraf, E. U. Avila, D. A. Genov, G. Bartal, and X. Zhang, Nature (London) 455, 376 (2008).

${ }^{6}$ A. Alù, A. Salandrino, and N. Engheta, Opt. Express 14, 1557 (2006); A. Alù and N. Engheta, Phys. Rev. B 78, 085112 (2008).

${ }^{7}$ D. S. Citrin, J. Opt. Soc. Am. B 22, 1763 (2005).

${ }^{8}$ L. D. Landau, E. M. Lifshitz, and L. P. Pitaevskii, Electrodynamics of Continuous Media (Pergamon Press, New York, 1984), Chap. 9.

${ }^{9}$ V. M. Agranovich, Y. R. Shen, R. H. Baughman, and A. A. Zakhidov, Phys. Rev. B 69, 165112 (2004).

${ }^{10}$ D. J. Cho, F. Wang, X. Zhang, and Y. R. Shen, Phys. Rev. B 78, 121101(R) (2008).

${ }^{11}$ V. L. Moruzzi, J. F. Janak, and A. R. Williams, Calculated Electronic Properties of Metals (Pergamon Press, New York, 1978), Chap. 4.

${ }^{12}$ M. Notomi, Phys. Rev. B 62, 10696 (2000); C. Luo, S. G.
Johnson, J. D. Joannopoulos, and J. B. Pendry, ibid. 65, 201104(R) (2002).

${ }^{13}$ A. Alù and N. Engheta, Phys. Rev. B 75, 024304 (2007); R. A. Shore and A. D. Yaghjian, IEICE Trans. Commun. E91-B, 1819 (2008).

${ }^{14}$ K. H. Fung and C. T. Chan, Opt. Lett. 32, 973 (2007).

${ }^{15}$ K. H. Fung and C. T. Chan, Phys. Rev. B 77, 205423 (2008).

${ }^{16}$ W. Y. Zhang, X. Y. Lei, Z. L. Wang, D. G. Zheng, W. Y. Tam, C. T. Chan, and Ping Sheng, Phys. Rev. Lett. 84, 2853 (2000).

${ }^{17}$ W. H. Weber and G. W. Ford, Phys. Rev. B 70, 125429 (2004).

${ }^{18}$ C. E. Dungey and C. F. Bohren, J. Opt. Soc. Am. A 8, 81 (1991).

${ }^{19}$ S. Y. Park and D. Stroud, Phys. Rev. B 69, 125418 (2004).

${ }^{20}$ Y. R. Zhen, K. H. Fung, and C. T. Chan, Phys. Rev. B 78, 035419 (2008).

${ }^{21}$ Y. Wu and Z. Q. Zhang, arXiv:0903.4799, Phys. Rev. B (to be published).

${ }^{22}$ D. R. Smith, S. Schultz, P. Markos, and C. M. Soukoulis, Phys. Rev. B 65, 195104 (2002).

${ }^{23}$ D. R. Smith and J. B. Pendry, J. Opt. Soc. Am. B 23, 391 (2006).

${ }^{24} \mathrm{P}$. Sheng, Introduction to Wave Scattering, Localization and Mesoscopic Phenomena (Springer, New York, 2006), Chap. 4.

${ }^{25}$ J. D. Jackson, Classical Electrodynamics, 3rd ed. (Wiley, New York, 1999).

${ }^{26}$ R. Merlin, Proc. Natl. Acad. Sci. U.S.A. 106, 1693 (2009).

${ }^{27}$ V. A. Markel, J. Opt. Soc. Am. B 12, 1783 (1995).

${ }^{28}$ X. D. Wang, X. G. Zhang, Q. L. Yu, and B. N. Harmon, Phys. Rev. B 47, 4161 (1993). 